EOMmun Communication et organisation

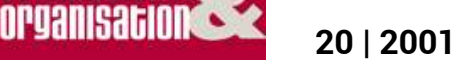

La communication du risque

\title{
Communication des risques dans les projets
}

\section{Hervé Courtot}

\section{OpenEdition}

Journals

Édition électronique

URL : http://journals.openedition.org/communicationorganisation/2553

DOI : 10.4000/communicationorganisation.2553

ISSN : $1775-3546$

\section{Éditeur}

Presses universitaires de Bordeaux

\section{Édition imprimée}

Date de publication : 1 novembre 2001

ISSN : 1168-5549

\section{Référence électronique}

Hervé Courtot, "Communication des risques dans les projets », Communication et organisation [En ligne], 20 | 2001, mis en ligne le 27 mars 2012, consulté le 19 avril 2019. URL : http://

journals.openedition.org/communicationorganisation/2553; DOI : 10.4000/

communicationorganisation.2553

Ce document a été généré automatiquement le 19 avril 2019.

(c) Presses universitaires de Bordeaux 


\title{
Communication des risques dans les projets
}

\author{
Hervé Courtot
}

1 Un grand nombre d'entreprises et de secteurs industriels ont cherché, ces dernières années, à appliquer le processus, les méthodes et les outils de management des risques dans leurs projets, poussés par :

2 - un environnement économique et industriel de plus en plus complexe, incertain et fluctuant qui perturbe les stratégies produits, les chiffrages économiques, les définitions techniques et les scénarios industriels, les plannings de réalisation et qui entraîne le plus souvent des ajustements coûteux et difficiles à réaliser ;

3 - une complexité accrue des projets, tant du point de vue technique, logistique, qu'organisationnel ;

4 - l'obligation de mieux maîtriser les coûts, les délais et les spécifications techniques des projets, vu la pression croissante des actionnaires privés ou publics, la médiatisation et l'impact de l'opinion publique, l'accentuation du contexte concurrentiel...

5 C'est ainsi que la prise en compte explicite des risques dans le déroulement des projets apparait aujourd'hui comme une nécessité pour les responsables de projet pour bien maîtriser leur projet et assurer leur réussite (en les incitant à plus de rigueur dans l'analyse du projet, en les forçant à anticiper les problèmes et à prévoir le plus en amont possible les dispositions nécessaires pour éviter qu'ils se produisent ou en atténuer leurs effets, en les conduisant à focaliser leurs efforts sur les points les plus sensibles...). Elle est devenue également un élément indispensable pour le développement des entreprises, voire pour leur pérennité (en évitant d'entreprendre des projets trop risqués ou mal conçus, en proposant un cadre méthodologique pour mieux maîtriser les risques détectés, en définissant des plannings et des budgets plus réalistes...).

6 Cependant, malgré l'intérêt et l'enthousiasme croissant que suscite dans les entreprises le management des risques des projets et la plus grande sensibilité des professionnels impliqués à la notion de risque, nous constatons que la communication dans ce domaine 
reste délicate et se trouve souvent entravée par un certain nombre de problèmes. Rendue responsable de bien des maux, la communication est souvent considérée comme trop lente à descendre ou à remonter, mal ciblée, mauvaise, inefficace, trop rare ou difficile à suivre, réservée à certains, contradictoire, mal coordonnée, etc. Aussi, l'objet de cet article n'est pas de décrire les principes, les méthodes et les outils utilisés pour gérer les risques d'un projet, ni de traiter de la problématique de la communication dans les organisations, il existe pour cela une littérature abondante, mais de rappeler que les enjeux du management des risques dans les projets sont essentiellement des enjeux de communication et que l'échange d'informations sur ce sujet est souvent difficile à réaliser. Pour cela, nous rappellerons tout d'abord brièvement les principales finalités de la communication dans le management des risques d'un projet. Nous nous attacherons ensuite à recenser les obstacles les plus couramment rencontrés. Enfin, nous terminerons en énonçant, de manière non-exhaustive et sans prétendre clore définitivement le sujet, quelques exigences à respecter pour remédier aux difficultés évoquées et garantir une communication rapide et efficace.

\section{Finalités de la communication dans le management des risques d'un projet}

7 Le management des risques d'un projet constitue une démarche rigoureuse qui classiquement vise à identifier, évaluer, hiérarchiser et maîtriser les risques encourus. Pour cela, elle requiert une communication bien préparée, organisée et administrée qui va permettre alors de :

- contribuer à définir de manière plus pertinente les différents objectifs du projet (en terme de délais, de coûts et de spécifications techniques), grâce à l'accroissement et à l'amélioration de la qualité des informations recueillies ;

9 - prendre en compte les divers imprévus, aléas et incertitudes susceptibles de survenir au cours du projet, et de fournir une meilleure connaissance de sa situation et de son niveau d'exposition aux risques; conduire à une meilleure maîtrise du projet, en ajustant son pilotage aux diverses évolutions de son environnement et en organisant la réactivité face aux divers événements susceptibles de se produire et de perturber son déroulement ;

10 - faciliter la prise de décision en fonction de l'urgence de la situation et de la faisabilité des actions de maitrise, d'obtenir une meilleure définition du niveau de priorité des actions à engager, et de concevoir le meilleur compromis possible ; accroître les chances de succès du projet, grâce à une meilleure compréhension et identification des risques encourus, et une meilleure définition des actions à entreprendre pour s'en prémunir.

11 Toutefois, il convient de souligner que le rôle de la communication ne se réduit pas, comme le rappellent Joly \& Muller (1994, p.119), à une simple transmission d'informations ou à un échange de données sur les événements redoutés et leurs conséquences. C'est également, la rencontre de personnes autour d'un sujet sur lequel chacun peut et doit s'exprimer. D'une part, elle facilite la mise en commun de connaissances, le partage de savoirs et d'expériences, contribuant ainsi à une augmentation des discussions et des confrontations d'idées et de points de vue. D'autre part, elle permet, grâce aux nombreux échanges indispensables à sa mise en œuvre et à son utilisation, d'obtenir de chacune des personnes impliquées dans le projet une prise de conscience partagée des risques encourus, un dialogue approfondi sur leurs causes et 
leurs conséquences, un partage d'informations pertinentes pour être a minima sensibilisés aux problèmes rencontrés, contribuant ainsi à créer le climat de confiance et de coopération indispensable pour la réussite du projet. Enfin, elle permet de mieux se connaître, d'apprendre à travailler en équipe, d'établir entre les différents professionnels mobilisés des relations efficaces et d'accroître leur interactivité avec un enrichissement mutuel, permettant ainsi de créer les conditions d'une collaboration fructueuse et une bonne compréhension de la situation, et d'obtenir un degré de consensus sur le niveau d'exposition au risque du projet.

\section{Obstacles à une bonne communication sur les risques d'un projet}

Bien que la communication joue un rôle primordial dans le management des risques d'un projet, bien qu'elle semble être aujourd'hui un vecteur efficace pour faire prendre les bonnes décisions et anticiper les problèmes éventuels, les observations que nous avons pu faire, ont révélé l'existence de plusieurs obstacles. Les difficultés les plus couramment rencontrées dans les projets résultent d'une part, du comportement des acteurs concernés (personnes, groupes, entités...), de leur système de valeur, de leurs habitudes, de leurs facteurs de motivation, de leur crainte devant l'incertitude... et d'autre part, des dispositifs de communication interne et externe mis en place.

\section{Les difficultés liées au comportement des individus}

Le management des risques d'un projet doit s'appuyer d'une manière générale sur une communication forte, des relations entre les personnes basées sur la transparence, la confiance réciproque, le droit à l'erreur. Elle exige de la part des personnes concernées une motivation, une collaboration et une implication de chacun. Or, nous constatons que l'attitude et le comportement des individus constituent dans de nombreux cas un obstacle à une bonne communication sur les risques, certaines informations étant biaisées, filtrées, amplifiées, camouflées. La communication dans ce domaine est souvent entravée par un certain nombre de problèmes provenant de :

14 - La répugnance qu'ont généralement responsables de projet à donner une image trop négative et pessimiste de la situation du projet pour ne pas démotiver et démobiliser les membres de l'équipe. Comme le soulignent Briner, Geddes \& Hastings, « la sensibilisation de son équipe aux risques du projet est un des actes les plus délicats du chef de projet, qui doit être à la fois prudent et audacieux, objectifs et soucieux de l'impact de ses propos ; s'il fait part de toutes ses craintes, il peut démotiver son équipe ; s'il les tait, on ne lui fera plus confiance » (1993, p. 9). En effet, la notion de risque, pris dans son assertion négative, est souvent considérée comme un obstacle, un danger, un dommage et provoque alors un sentiment de peur et de crainte inspiré par cette culture d'aversion pour le risque qui prédomine encore aujourd'hui.

15 - Le comportement de fuite (peur de dire des âneries, volonté de ne pas paraître naïf ou insensé) ou de mépris (refus d'accepter des opinions émises par des personnes ayant un statut différent, tendance à juger ou à critiquer tout ce qui est dit...) de certains participants lors de la mise en évidence des risques ou de leur traitement. 
- Le fatalisme et la passivité de certains acteurs-projet. Face aux difficultés généralement rencontrées pour recenser et caractériser de manière exhaustive les risques encourus, pour évaluer leur niveau de criticité ou pour définir et mettre en place des parades efficaces, il est fréquent de voir des acteurs d'un projet avoir la tentation de se réfugier dans une attitude d'attente et de passivité (« on verra bien ce qui va se passer!» et accepter, avec un fatalisme, que des événements dommageables, qu'ils n'avaient pas pris en compte ou pas suffisamment dans leur analyse et leur synthèse des risques du projet, puissent influer notablement sur son bon déroulement, voire sur son succès (« de toute façon on n'y peut rien ! »...).

- La tentation à occulter ou à minimiser certains risques, à ne pas parler des problèmes rencontrés ou des erreurs commises, des oublis ou des confusions faites... soit pour éviter l'émergence de conflits de pouvoirs, soit pour éviter de s'attirer les foudres de la hiérarchie (peur de la sanction, crainte de voir le projet être remis en cause, voire abandonné), soit pour ne pas montrer son manque de compétences ou son incompétence à bien maîtriser le projet. Une bonne communication doit prendre en compte tous les faits marquants, même s'ils dérangent. Or, nous constatons que l'apparition de dérives ou de dysfonctionnements se traduit généralement plus par une recherche systématique du (es) responsable(s), que par une analyse causale et une recherche approfondie des parades à engager. Cette réflexion rejoint celle exprimée par Declerck, Emery et Crener (1980, p. 43) pour qui « lorsqu'on étudie les causes de ces retards, c'est généralement pour trouver le responsable et il est rare que l'on obtienne ainsi des renseignements valables sur les véritables carences du processus d'exécution. En fait, il s'agit le plus souvent de trouver un bouc émissaire, de rejeter la responsabilité sur quelqu'un d'autre, de se défendre et de trouver des excuses ».

- La méfiance envers ceux que l'on ne connait pas. La réalisation d'un projet nécessite l'intervention de plusieurs acteurs, d'origines, de cultures, de métiers, de fonctions, de directions ou d'entreprises différentes, et qui dans la plupart des cas ne se connaissent pas, n'ont jamais eu l'occasion de travailler ensemble avant le projet et œuvrent pour des objectifs différents, voire opposables. Aussi, il est fréquent d'observer une méfiance à transmettre convenablement et rapidement les informations détenues aux différentes parties prenantes, qui s'explique par une crainte des jugements, par la peur que des personnes profitent de la situation, par la nécessaire confidentialité des informations... De nouveaux interlocuteurs apparaissent, qu'il convient d'apprendre à connaître et dont il faut comprendre les habitudes de travail, les exigences et les canaux d'information qui répondront à ces besoins.

- La «rétention d'information». Une des difficultés rencontrées en matière de communication sur les risques est la conservation des informations comme source de valorisation et de pouvoir. En effet, chaque individu détenant ou ayant la capacité d'obtenir des informations utiles pour les autres, il arrive fréquemment de voir des personnes les conserver plus ou moins jalousement considérant que ce sont des facteurs essentiels de valorisation, de pouvoir informel et de responsabilité ou parce qu'elles représentent pour elles une marge d'autonomie par rapport à l'organisation. 


\section{Les difficultés liées aux dispositifs de communication mis en place et à la qualité des informations échangée} de communication interne et externe adéquats et performants, afin de maintenir l'intérêt, l'enthousiasme et l'adhésion des différents intervenants. Or, nous constatons que les informations diffusées sont souvent mélangées, redondantes, incomplètes, difficilement accessibles... La bonne circulation de l'information représente un enjeu majeur. Ceci dit, la communication sur les risques est souvent entravée par :

21 - La dispersion géographique des sites et l'éclatement des intervenants au sein des directions ou services qui peuvent gêner ou empêcher ainsi la tenue de réunions périodiques ou circonstancielles (groupes de réflexion, interviews d'experts, réunions de suivi du portefeuille des risques ou de reporting...) pour traiter des risques.

- Le cloisonnement des directions et des services impliqués dans le projet. Dans de nombreux cas, la limitation des échanges d'information s'explique par le cloisonnement traditionnel des métiers ou de l'incommunicabilité naturelle ou les rivalités qui existent entre certains services ou certaines fonctions. De même, certains directions ayant la volonté d'exercer un contrôle fort sur les risques détectés, ceci nuit au développement de circuits de communications fluides et efficaces.

23 - L'excès de procédures et les lourdeurs bureaucratiques. De nombreux blocages ou lenteurs dans les échanges d'informations résultent de la mise en place d'un trop grand nombre de procédures formelles et de règles, de lourdeurs bureaucratiques, de la multiplicité des niveaux hiérarchique dans la structure de reporting... obligeant les acteurs à utiliser des réseaux professionnels officieux plutôt que les circuits codifiés de l'organisation.

24 - L'inexistence ou l'inefficacité des procédures et des outils de communication utilisés pour rassembler et conserver les divers types d'information sur les risques. Malgré l'essor de nouvelles technologies de l'information et de la communication (groupeware, GED et workflow, logiciels spécifiques, site Internet/Intranet, courrier électronique...), un certain nombre de problèmes constatés proviennent de l'inexistence ou de l'inefficacité des procédures ou des outils de communication utilisés : rapports écrits trop volumineux, mal structurés ou incomplets, réunions d'analyse et de suivi superflues, fichiers électroniques ou informatiques inexploitables, banque de données difficilement accessibles, destinataires noyés sous un amoncellement de documents ou de messages électroniques... Souvent séduites par la multitude d'instruments et d'outils de communication nouveaux qui s'offrent à elles, les entreprises ne prennent pas toujours le temps de réfléchir à la définition des objectifs de communication qui doivent présider au choix de ces outils.

- Le faible retour d'expériences. L'analyse que nous avons pu mener sur quelques projets, nous amène à constater aujourd'hui que non seulement la capitalisation des connaissances et des expériences acquises est souvent partielle, voire même totalement ignorée, mais également que leur transmission et leur diffusion est souvent insuffisante (Courtot, p.110). Elle n'a d'intérêt que si elle est collective et pas uniquement individuelle. Or, nous nous apercevons souvent que les moyens nécessaires à assurer une bonne capitalisation ne sont pas toujours développés ou utilisés, et quand ils le sont, ils ont souvent plus une dimension individuelle que collective. Cette observation rejoint

Communication et organisation, 20 | 2012 
celle faite par Midler pour qui «la mise en commun des expériences n'est pas quelque chose de spontané et naturel » (1993, p. 188).

\section{Quelques exigences à respecter pour remédier aux difficultés évoquées}

Pour remédier aux difficultés évoquées et garantir la mise en œuvre d'une communication rapide et efficace sur les risques encourus par un projet, il convient alors de respecter quelques exigences, à savoir :

- Une transparence, une réactivité, une solidarité et une confiance réciproque entre les acteurs. Aux dires de plusieurs responsables de projet, la transparence entre les divers intervenants est un élément essentiel pour une bonne gestion des risques du projet, car elle contribue à instaurer un climat de confiance et de collaboration.

- La mise en place de systèmes d'information pertinents, efficaces et adaptés, permettant de disposer de données mises à jour régulièrement et disponible à tout moment, tout en respectant les méthodes de travail et les cultures métiers. Il est important de permettre à chacun d'avoir une vision globale et réaliste de la situation du projet (état d'avancement du projet, retards ayant un impact, modifications apportées, problèmes ou solutions techniques risquant d'affecter la façon de conduire le projet...), au lieu de s'enfermer dans son domaine d'activité, sa discipline ou sa spécialité. Comme le rappelle le PMI, « il faut prendre soin d'éviter un gaspillage des ressources par des informations inutiles et une technologie inappropriée " (1998, p. 163). L'information constitue la matière première pour bien maîtriser les risques d'un projet. Aussi, le concept de risque étant étroitement associé à celui d'information, si l'information est insuffisante, l'incertitude est plus grande, d'où un risque plus important. Au contraire, lorsqu'on dispose suffisamment d'informations pertinentes, l'incertitude est moindre, d'où une diminution du risque.

- Un engagement clair et soutenu de la hiérarchie dans la démarche. Il est important que les dirigeants manifestent leur implication et leur intérêt pour le management des risques des projets en fournissant les ressources et les moyens nécessaires et en favorisant la prise de responsabilité, la délégation de pouvoirs, l'autonomie, le volontarisme et le goût pour le risque. Le besoin essentiel des acteurs est d'être compris et soutenus dans leurs actions. Mais, encore faut-il que la hiérarchie résiste à la tentation de demander plus d'explications, de faire un contrôle trop tatillon, d'influer ou de critiquer régulièrement les décisions prises, de remettre en cause certaines solutions envisagées et les critères ayant présidé aux choix de la parade retenue... sous peine de créer un climat de méfiance, de démotivation et de fausser ainsi les informations remontées. Il est important d'éviter que les questionnements sur les risques ne soient ressentis comme une ingérence ou un flicage.

30 - Le développement d'une culture risque où tous les participants reconnaissent qu'ils sont dans une certaine mesure gestionnaires des risques, où toutes les personnes disposent d'une réelle capacité d'influence et d'action, où chacun prend l'habitude de parler de risques, où il est reconnu une forme de droit à l'erreur.

31 - La formalisation et le partage des connaissances et des expériences passées en matière de gestion des risques. La connaissance historique des incidents rencontrés (origines, caractéristiques, impacts...), des décisions prises, des parades choisies et leurs coûts associés, la recommandation sur l'efficacité des méthodes et outils de gestion des 
risques... sont fondamentales pour empêcher les tâtonnements, la reconduction de développements inutiles et la reproduction d'erreurs et de dysfonctionnements. Elles permettent d'enrichir la connaissance des risques potentiels et dommageables, d'accroître la réactivité à chaque niveau d'intervention, de renforcer les nouvelles pratiques, de faciliter la prise de décision et d'améliorer l'efficacité des actions de maîtrise.

- Une clarification des responsabilités. Le management des risques d'un projet est une démarche collective au sens où elle associe tous les acteurs, et individuelle au sens où chaque acteur est impliqué dans le cadre d'une responsabilité déterminée. Il est donc important de bien définir et documenter les responsabilités de la gestion des risques liés à un projet et les interfaces avec d'autres fonctions, pour éviter l'ambiguïté quant au partage des rôles (" qui fait quoi ? ») et l'incompréhension des missions de chacun (« qui décide de ce qu'il y a à faire?»). Pour chaque risque à traiter, il convient de s'assurer qu'une personne soit désignée en tant que responsable de cette activité de traitement. En effet, il est important que chacun des participants (dirigeants, directeurs métiers, chefs de projet, partenaires...) ait une idée claire de ce que l'on attend d'eux, de leur place dans le dispositif, et des personnes à qui s'adresser pour obtenir des précisions. Quand chacun sait quelles sont ses responsabilités propres, la communication peut alors s'établir.

\section{Conclusion}

Bien que le management des risques dans les projets soit à la mode et que de nombreuses entreprises s'accordent sur son intérêt et revendiquent de mettre en œuvre ses principes et ses méthodes, notre expérience d'accompagnement de nombreux projets nous amène à constater un décalage entre les espérances et la réalité des résultats. Communiquer c'est facile, mais bien communiquer c'est plus difficile. L'inexistence d'informations explicites et fiables sur les risques constitue un handicap majeur se traduisant souvent par une mise devant le fait accompli, imposant alors de traiter le problème de façon urgente et rapide. Plus les risques sont détectés tardivement, plus leurs conséquences peuvent être graves et moins les solutions à mettre en œuvre seront négociables. En effet, de nombreux projets échouent en raison d'une communication sur les risques mal ou insuffisamment préparée, organisée et administrée. L'efficacité du management des risques dépend non seulement de la qualité des échanges d'information et de l'organisation des actions de communication, mais également de la qualité relations entre les parties en communication. Il est donc essentiel pour la mise en œuvre de ce type de démarche, voire pour la réussite du projet, de détecter, d'analyser et de résoudre les problèmes de communication, et de veiller à ce que cette communication s'effectue rapidement et efficacement. 


\section{BIBLIOGRAPHIE}

BRINER, W., GEDDES, M. \& HASTINGS, C, Le manager de projet : un leader, Édition Afnor Gestion, 1993, $177 \mathrm{p}$.

COURTOT, H., La gestion des risques dans les projets. Édition Economica, 1998, 294 p.

DECLERCK, R. P., EMERY, P. \& CRENER, M. A., Le Management stratégique des projets. Éditions

Hommes et Techniques, 1980, 198 p. JOLY, M. \& MULLER, J-L., De la gestion de projet au management par projet. Édition Afnor, 1994, 213 p.

MIDLER, C, L'auto qui n'existait pas. InterEditions, 1993, 203 p.

PMI, Management de projet - Un référentiel de connaissances. Édition Afnor, 1998, 266 p.

\section{RÉSUMÉS}

L'objet de cet article est de rappeler, sur la base d'un retour d'expérience issu de l'industrie et de l'ingénierie, que les enjeux du management des risques dans les projets sont essentiellement des enjeux de communication et que la communication dans ce domaine reste délicate et difficile à réaliser en raison d'un certain nombre d'obstacles issus du comportement des acteurs mobilisés, mais aussi des dispositifs de communication mis en place. Aussi, pour garantir une communication rapide et efficace, il convient de respecter certaines exigences. L'efficacité du management des risques dépend non seulement de la qualité des échanges d'information et de l'organisation des actions de communication, mais également de la qualité relations entre les parties en communication.

The object of this article is to recall, on the basis of experience feedback resulting from industry and engineering, that the stakes of the risk management in the projects are essentially stakes of communication and that the communication in this field remains delicate and difficult to realize because of a certain number of obstacles resulting from the behavior of the mobilized actors, but also of the devices of communication set up. So to guarantee a fast and effective communication, it is advisable to respect certain requirements. The efficiency of the risk management depends not only on the quality of the exchanges of information and the organization of the actions of communication, but also of the quality of the relations between the parts in communication.

\section{INDEX}

Mots-clés : gestion des risques, management de projet, communication du risque

\section{AUTEUR}

\section{HERVÉ COURTOT}

À la fois chercheur, enseignant et consultant en Management de projet et en Management des risques dans les projets, Hervé Courtot intervient au sein de la société France 
Télécom, à la Direction Qualité des Fournisseurs (DQF), en tant que responsable de l'activité conseil en « Qualité des Projets ». Docteur ès Gestion, il est l'auteur d'un ouvrage sur « La gestion des risques dans les projets », paru aux Éditions Economica, et de plusieurs articles et communications sur le sujet. 